\title{
CHERPAC, an environmental transport code, and its predictions of environmental sensitivities in agricultural and forest ecosystems
}

\author{
S.L. Chouhan ${ }^{1}$, N.W. Scheier ${ }^{1}$ and S.-R. Peterson ${ }^{2}$ \\ ${ }^{1}$ Environmental Technologies Branch, Atomic Energy of Canada Limited (AECL), \\ Chalk River, Ontario, KOJ 1JO, Canada, \\ ${ }^{2}$ Retired from $A E C L$ \\ e-mail: Chouhans@aecl.ca
}

\begin{abstract}
CHERPAC is a code developed by AECL to predict the time-dependent concentrations of 25 radionuclides in environmental compartments and the resulting radiation dose (ingestion, inhalation, immersion and groundshine) to humans, following an accidental release to the atmosphere from a nuclear facility. CHERPAC was used to investigate environmental sensitivities in agricultural and forest ecosystems. Given the assumptions in the CHERPAC code, it was concluded that doses to humans from agricultural food products are higher than those from groundshine and forest food products. Doses from agricultural products are highest from radionuclide deposition in summer because all plants are at their peak growth and are ingested fresh after the deposition event. The dose is higher if the deposition occurs in dry conditions rather than during heavy rain, because radionclides adhere better to dry plant leaves. For Cs-137, ingestion dose is higher for adults than other age groups, but for Sr-90 and I-131, ingestion dose is highest for infants. This is due to relative food product concentrations, intake rates and DCFs (dose conversion factors). In the forest ecosystem, adult doses are higher than those for children and infants because of the higher rates of intake by adults of forest food products.
\end{abstract}

\section{INTRODUCTION}

CHERPAC (Chalk River Environmental Research Pathways Analysis Code) is a code developed by AECL to predict the time-dependent concentrations of 25 radionuclides in environmental compartments and the resulting radiation doses (from ingestion, inhalation, immersion and groundshine) to humans, following an accidental release to the atmosphere from a nuclear facility. CHERPAC was developed and improved by testing in international model intercomparison studies using Chernobyl fallout data. Some sub-models, and parameter values for them, were originally taken from a Canadian routine-release dose calculation model and adapted for simulating accidental releases. The pathways for environmental radioactivity transport and human exposure that are modeled in CHERPAC are shown in Figure 1. The code accounts for seasonality and can be used for an accident occurring at any time of the year. It also accounts for decay and buildup during the time between the harvest of food products and their ingestion, as well as losses resulting from food processing. CHERPAC can make stochastic predictions using a Latin hypercube sampling method, and sensitivity analyses are carried out using partial correlation and standardized regression coefficients.

\section{USING CHERPAC TO INVESTIGATE ENVIRONMENTAL SENSITIVITIES}

CHERPAC was used to investigate environmental sensitivities for a scenario in the IAEA (International Atomic Energy Agency) EMRAS-II (Environmental Modelling for Radiation Safety- Phase II) project. In this scenario, a hypothetical instantaneous deposition of $1000 \mathrm{~Bq} \mathrm{~m}^{-2}$ of Cs-137, Sr-90 and I-131 was assumed to occur. Cases of agricultural and forest ecosystems were considered. People living in 


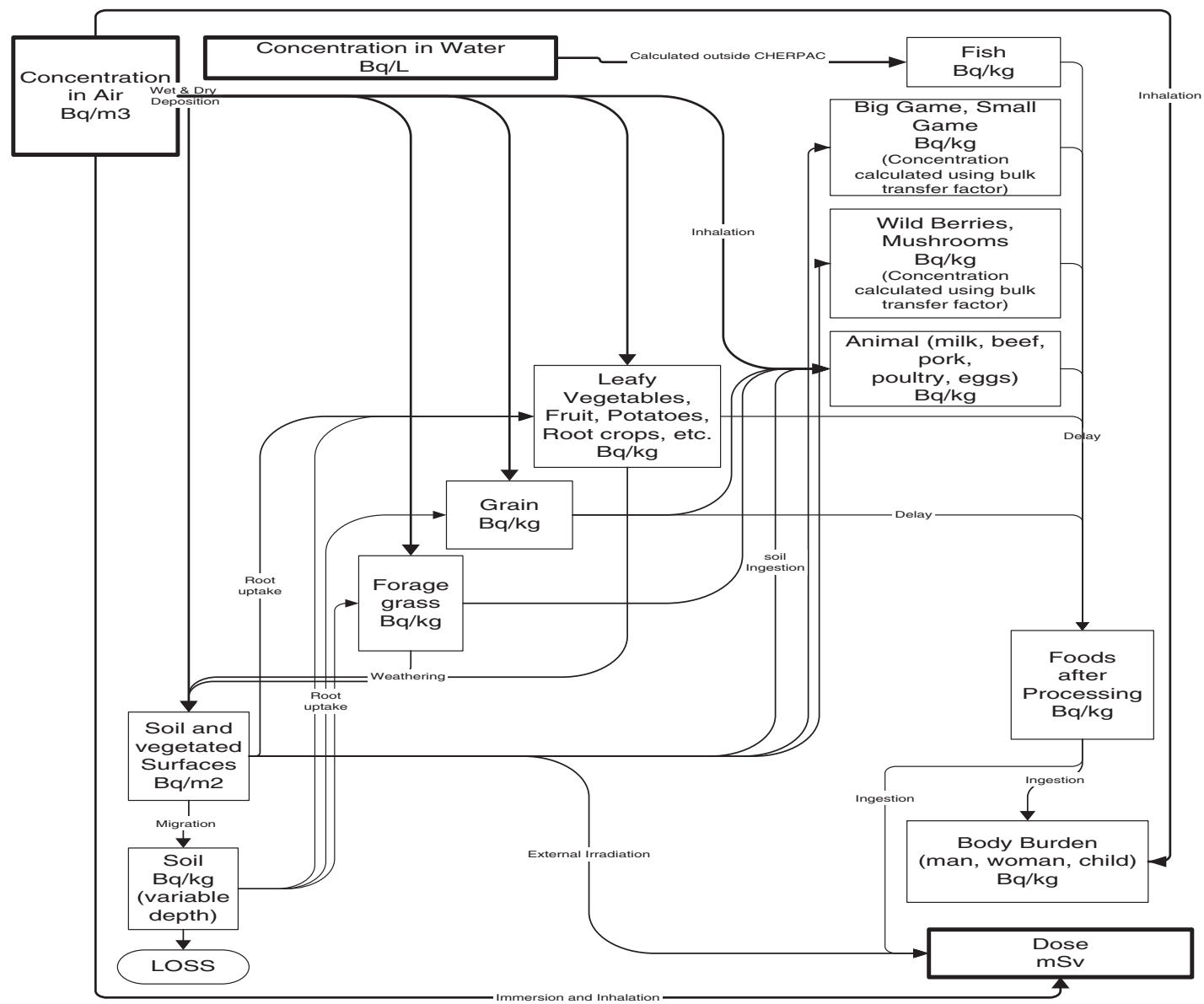

Figure 1. Pathways modelled in CHERPAC.

the forest ecosystem were assumed to consume uncontaminated agricultural food products and vice versa. Cases of deposition under dry or heavy rainfall conditions were evaluated. Seasonal effects were evaluated by considering cases in which the deposition occurred in winter, spring, summer, or autumn. The concentrations in common food products and doses to an adult, a child (10 year old), and an infant (1 year old) were predicted for a two-year period following the deposition. Calculations were performed using CHERPAC's default parameter values, which are mostly specific to Ontario, Canada. All radionuclides were assumed to be deposited in particulate form.

\subsection{Results: time-dependent concentrations and doses: Cs-137, dry deposition in summer (August)}

Predicted accumulated activity in top mixed layer of vegetated soil:

- The activity is $\sim 300 \mathrm{~Bq} / \mathrm{m}^{2}$ in the first month, peaks at $\sim 1000 \mathrm{~Bq} / \mathrm{m}^{2}$ in the fourth month, and then slowly drops to $\sim 900 \mathrm{~Bq} / \mathrm{m}^{2}$ at the end of 2 years as a result of radioactive decay and loss to deeper soil.

- Soil activity is low in the first 3 or 4 months because activity is retained by the plant leaves. Later on, the activity on the leaves gradually washes off and transfers to soil. 


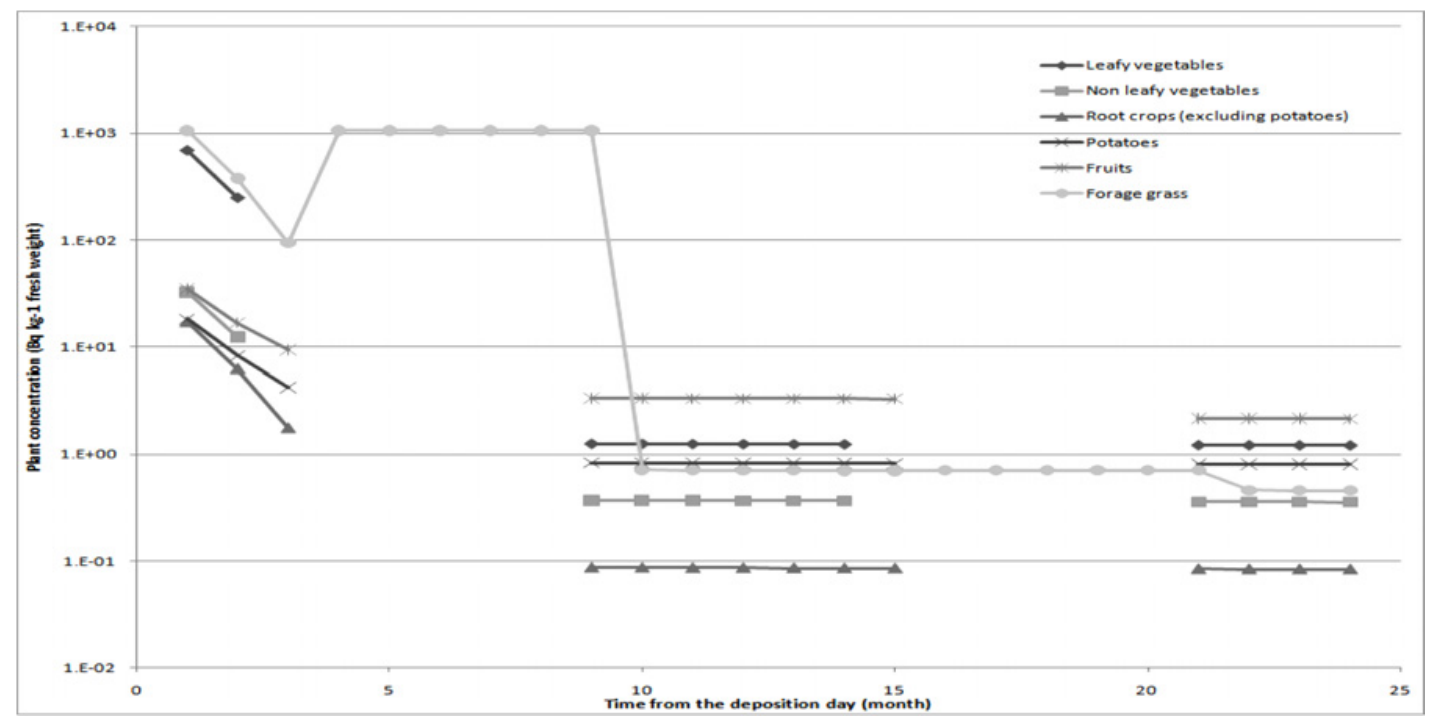

Figure 2. Predicted concentrations in agricultural plants: Cs-137, dry deposition in summer (August).

- More activity is initially retained on the plants if the deposition occurs in dry conditions rather than in heavy rain, because radionuclides adhere better to dry plant leaves.

- Soil activity is also sensitive to the timing of the deposition. For example, if deposition occurs close to harvest, then some deposited activity is removed by cropping, which reduces the activity in the soil.

Predicted concentrations in agricultural plants (Figure 2):

- Concentrations are highest in forage grass and leafy vegetables because these plants have parameter values that favour the uptake and retention of radionuclides.

- The forage grass concentrations shown here are those for grass ingested by dairy cows. Dairy cows eat fresh grass from May to October and grass harvested in August from November to April. Thus the concentration in forage in month 4 (November) increases to the August concentration.

- Activity deposited directly on the plants is lost with a removal half-life of 15 days.

- Plant concentrations are dependent on the plant rooting depth and the yearly migration of activity.

Predicted concentrations in domestic animal products (Figure 3):

- No radioactivity is predicted in beef for the 2 months after the deposition because the cattle are assumed to eat stored grass from the previous year. The concentration rises when they start eating grass harvested in the current year.

- Dairy cows are assumed to eat fresh grass in the first 3 months after deposition, and the activity in milk and cheese decreases with the decrease in forage concentration in this period. Predicted milk and cheese concentrations increase in November when the cows start eating stored grass.

- In the first year, predicted concentrations in chickens and eggs build up only through soil ingestion, because chickens are assumed to eat grain harvested before the deposition. When chickens are taken indoors in November, the concentration in eggs drops to zero immediately, and the concentration in chicken meat drops to zero within the 3-month life span of the chickens.

- The predictions for pigs are for Canadian animals that are kept indoors. Slaughter is assumed to occur 6 months after birth. For the first 2 months, pigs drink milk, and for all 6 months they eat grain. 


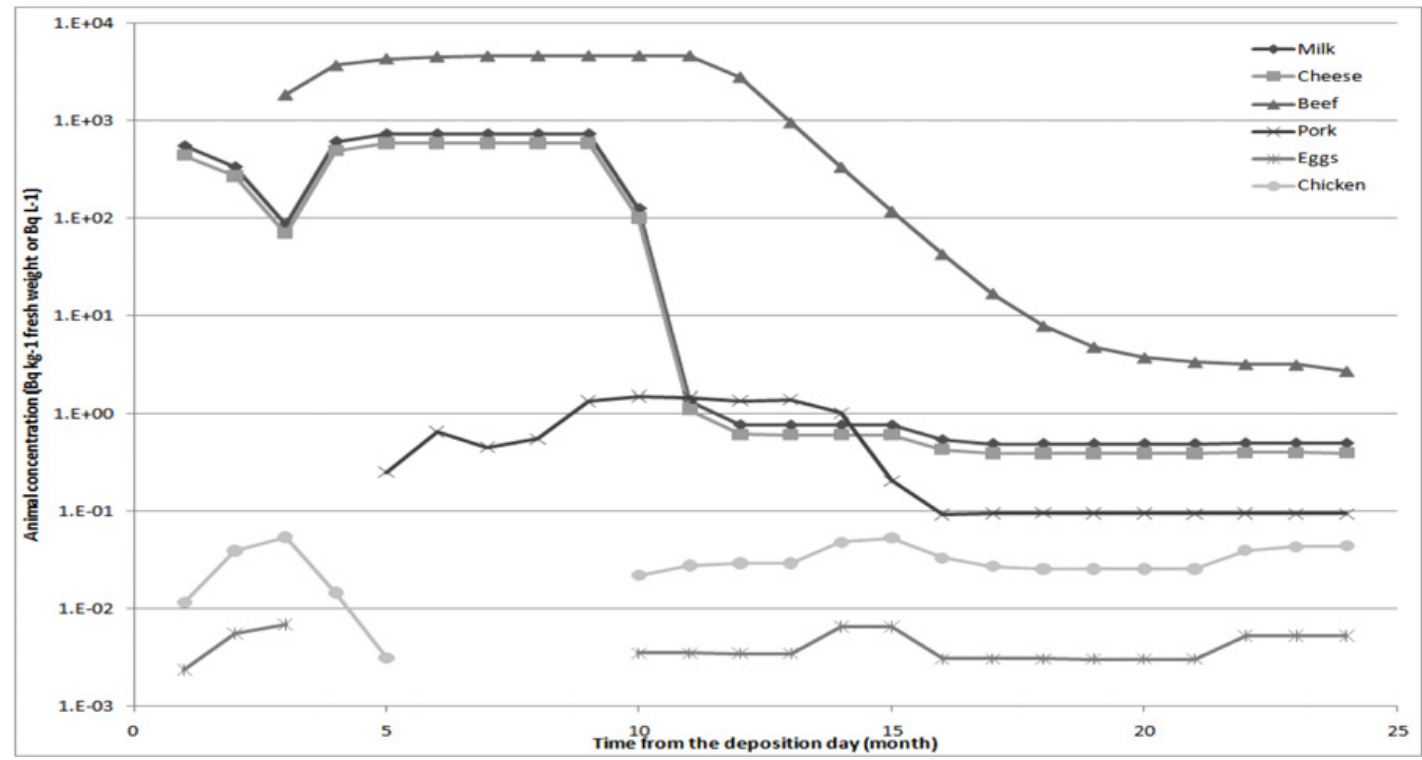

Figure 3. Predicted concentrations in domestic animal products: Cs-137, dry deposition in summer (August).

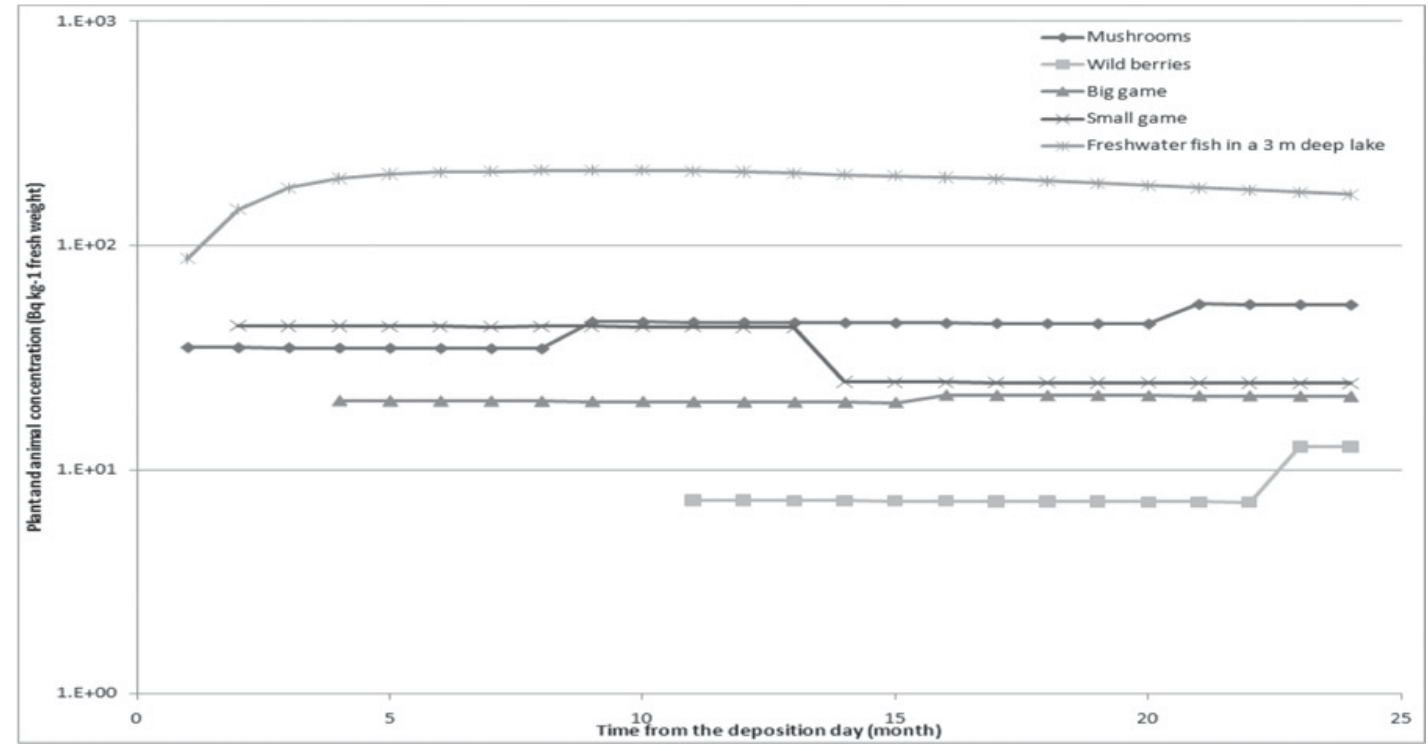

Figure 4. Predicted concentrations in forest food products: Cs-137, dry deposition in summer (August).

\section{Predicted concentrations in forest food products (Figure 4):}

- Concentrations in all products except fish are based upon bulk transfer factors observed after the Chernobyl accident.

- The concentration in mushrooms increases slightly and then drops as the modelled activity passes through the root zone.

- There is no activity predicted in wild berries for the first 10 months because deposition was assumed to occur later in the year than the berry harvest. 


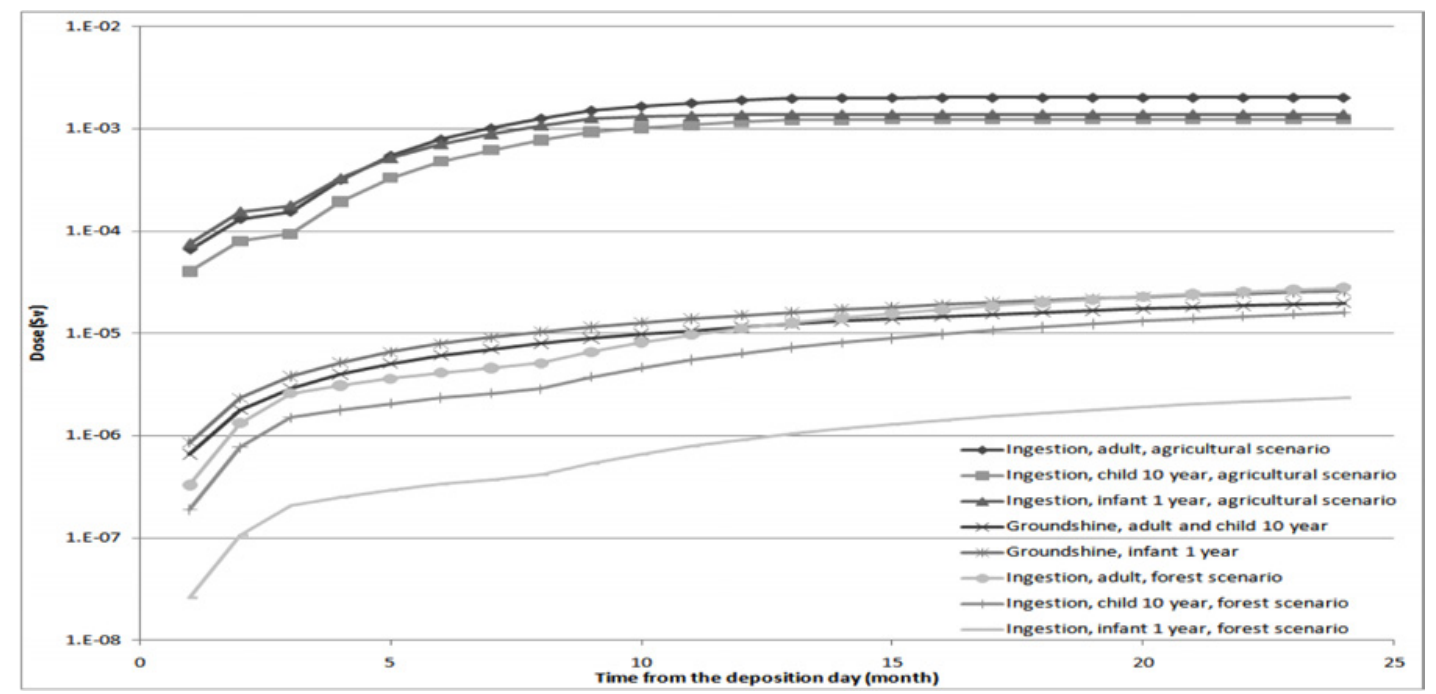

Figure 5. Predicted doses to humans: Cs-137, dry deposition in summer (August).

- Differences in concentrations in big and small game are due to the assumptions about foods consumed.

- Concentrations are higher in fish than in other food products because of the relatively high bioaccumulation factor for fish.

Predicted doses to humans (Figure 5):

- Ingestion doses from agricultural food products are higher than those from food products from forests or lakes.

\subsection{Results: final doses at the end of two years.}

Results shown in Figure 6 are ingestion doses from Cs-137, I-131, and Sr-90 to adult, child, and infant at the end of two years for the agricultural ecosystem with dry deposition occurring at different times of the year. More detailed results and doses predicted for other scenarios (e.g. from wet deposition, forest food products and groundshine) will be published by the IAEA.

Effect of radionuclide: Different radionuclides impart different doses because they have different deposition properties, transfer rates, accumulation rates, DCFs and half-lives. For example, because of its short half-life, the dose from I-131 from deposition in the fall or winter is significantly lower than doses from Cs-137 and Sr-90, which, because of their long half-lives, exhibit similar doses regardless of season of deposition.

Effect of deposition month: Predicted doses from the agricultural pathways are highest if deposition happens in summer (August), and lowest if it happens in fall (November). For summer deposition, the plants are nearly ready to be harvested, whereas no plants are growing in the fall, and a lot of activity is lost from the soil before the ground is seeded in the spring.

Effect of deposition type: For agricultural ecosystems, if the deposition occurs in spring or summer, the ingestion dose under dry conditions is up to three times higher than that under heavy rainfall conditions, because radionuclides adhere better to dry plant leaves.

Effect of age group: For the agricultural pathways, the dose from Cs-137 is highest for adults, whereas the doses from Sr-90 and I-131 are highest for infants. This is because of the relative food product concentrations, intake rates and DCFs. The relative groundshine doses to different age groups depend mainly upon the relative DCFs. 


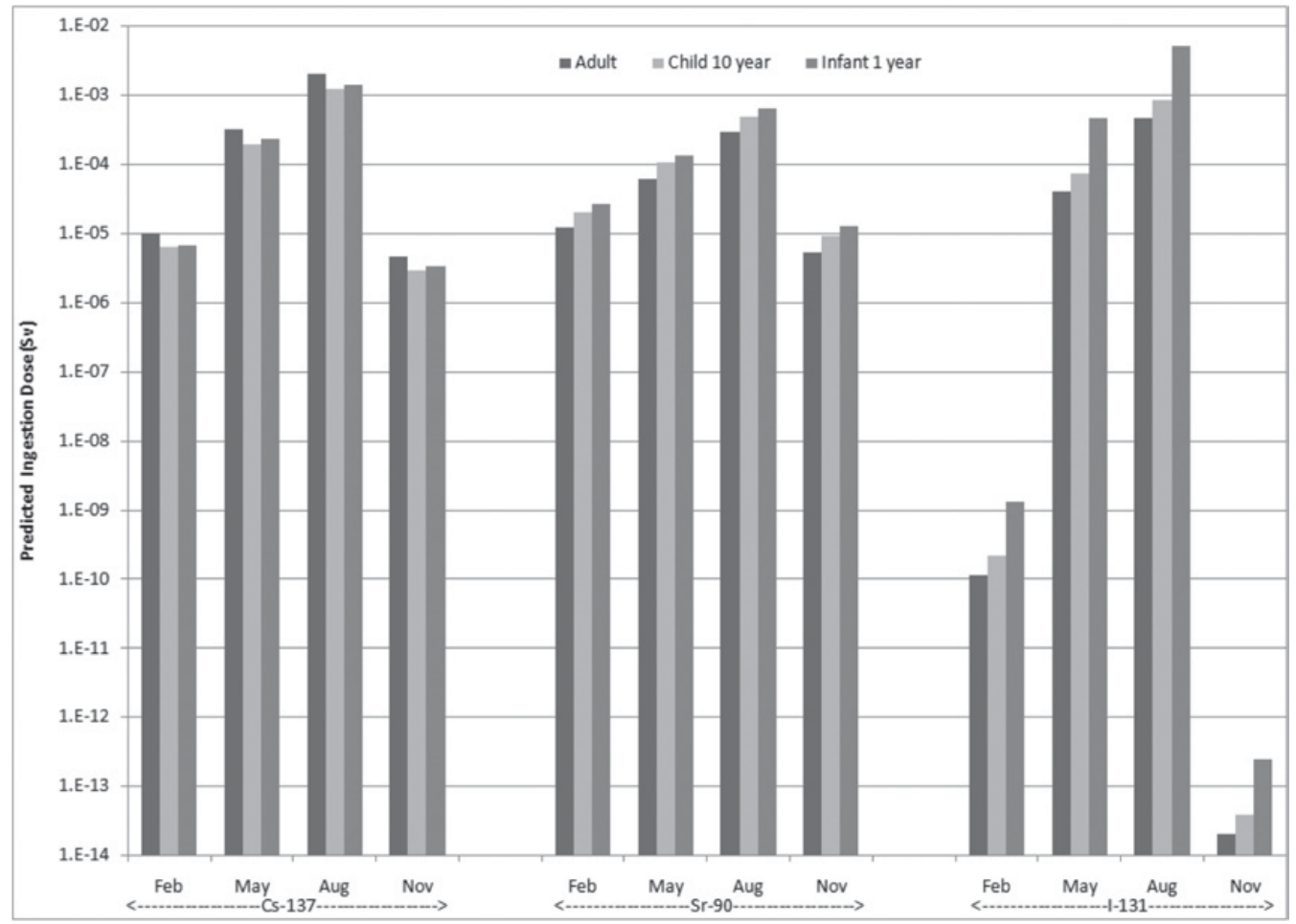

Figure 6. Predicted ingestion dose at the end of two years: dry deposition, agricultural ecosystem, four deposition months.

Effect of exposure pathway: Ingestion pathways give more dose if the deposition occurs in the spring or summer months than that if the deposition occurs in the fall or winter months. Dose from the groundshine exposure pathway is not season dependent.

Effect of Ecosystem: Humans living in an agricultural ecosystem will receive higher doses than those living in a forest ecosystem.

\section{CONCLUSIONS}

In this paper, CHERPAC, a time-dependent environmental transport model, was introduced. Based on its predictions, it was concluded that doses to humans from agricultural food products are higher than those from groundshine and forest food products. Doses from agricultural products are highest from radionuclide deposition in summer because all plants are at their peak growth and are assumed to have been ingested fresh after the deposition event. The dose is higher if the deposition occurs in dry conditions rather than during heavy rain, because radionclides adhere better to dry plant leaves. For Cs137, the ingestion dose is higher for adults than other age groups, but for Sr-90 and I-131, the ingestion dose is highest for infants. This is a result of relative food product concentrations, intake rates and DCFs. In the forest ecosystem, adult doses are higher than those for children and infants because of assumed higher rates of intake of forest food products by adults. 